P4 ISOLATED MEDIASTINAL LYMPH NODE TUBERCULOSIS (IMLNTB) IS CHARACTERISED BY ELEVATION IN SYSTEMIC AND BRONCHIAL IL-12 PATHWAY MEDIATORS COMPARED TO PULMONARY TB

H Jarvis, RS Thwaites, T Tunstall, JN Nanan, M Tolosa-Wright, I Marwah, AK Reuschl, TT Hansel, A Lalvani, OM Kon. Imperial College London, London, UK

10.1136/thoraxjnl-2017-210983.146

Introduction/Aims The incidence of isolated mediastinal lymph node tuberculosis (IMLNTB) has increased in recent years in the UK. These patients exhibit a distinct clinical phenotype compared to patients with pulmonary TB (PTB), more likely to be asymptomatic or presenting with immune hypersensitivity. The mediastinal lymph nodes are of particular interest in TB as they have been implicated as a key site for host-pathogen interaction, the outcome of which determines infection status within the dynamic spectrum of disease. Indeed, studies have suggested that IMLNTB may represent a sub-clinical phenotype with a greater degree of immune containment compared to PTB. This study assessed immune status in patients with IMLNTB, PTB and healthy controls with the hypothesis that that these states vary in levels of protective immunity.

Methods A novel sampling technique using a synthetic absorptive matrix (bronchosorption) placed on the respiratory mucosa of the bronchus was used to obtain mucosal lining fluid (MLF) of patients undergoing bronchoscopy. Serum samples were also collected in addition to clinical, radiological and demographic data. Eluted bronchosorption fluid, together with serum, were then analysed for a range of soluble inflammatory mediators using a multiplex immunoassay platform.

Results Patients with IMLNTB $(n=12)$ had elevated levels of IL-12/IL-23-p40 in both the serum and bronchial MLF compared to patients with PTB $(n=12)$ and healthy controls $(n=19)$. In addition, IL-12 induced IFNy pathway mediators, including TNF- $\alpha$, were elevated in the serum in the IMLNTB group. Conversely, levels of serum acute phase reactants (CRP/ SAA) were elevated in PTB compared to IMLNTB and healthy controls.
Conclusion These Results suggest that IMLNTB and PTB have different molecular phenotypes, with IMLNTB showing less systemic inflammation in the form of serum CRP/SAA, but greater or equal levels of levels of certain immune mediators in both the serum and the bronchus. These findings may reflect that IMLNTB is a distinct clinical state to PTB, with immune activation through the IL-12 pathway playing a role in achieving immune containment.

\section{P5 PLEURAL TUBERCULOSIS IN LONDON: A PERSISTENT DIAGNOSTIC CHALLENGE}

L Peters, GK Russell, LJ Martin, LY Han, M O'Donoghue, OM Kon, CL Ross. Imperial College Healthcare NHS Trust, London, UK

\subsection{6/thoraxjnl-2017-210983.147}

Background Five percent of disease due to Mycobacterium tuberculosis is reported to be associated with a pleural effusion. We conducted a retrospective review of all patients at our institution treated for pleural tuberculosis (TB) between 2010 and 2015, reviewing demographics, fluid microbiology, histology, and radiology to characterise our cohort and review diagnostic certainty.

Results 71\% (36/51) of our cohort were male; with a mean age of 41 years. $59 \%$ of cases had clear evidence of concurrent parenchymal involvement (five did not have cross sectional imaging). All effusions were exudates. 46 patients had imaging one year after treatment started. 16/46 had residual fluid, 16/46 demonstrated complete resolution and 14/46 had pleural thickening. Pleural fluid TB culture was performed in $47 / 51$ patients and was positive in $43 \%$ of cases. Pleural fluid TB PCR was performed in $18 / 51$ patients. $3 / 18$ were PCR positive; in 1 patient PCR provided a diagnosis 10 days before culture. Two PCR Results provided diagnoses despite negative cultures and demonstrated a rifampicin resistance mutation in one case. 21 pleural biopsies were done, 13 were video assisted thoracoscopic procedures and 8 were taken with closed cutting needles. Pleural biopsy TB culture was

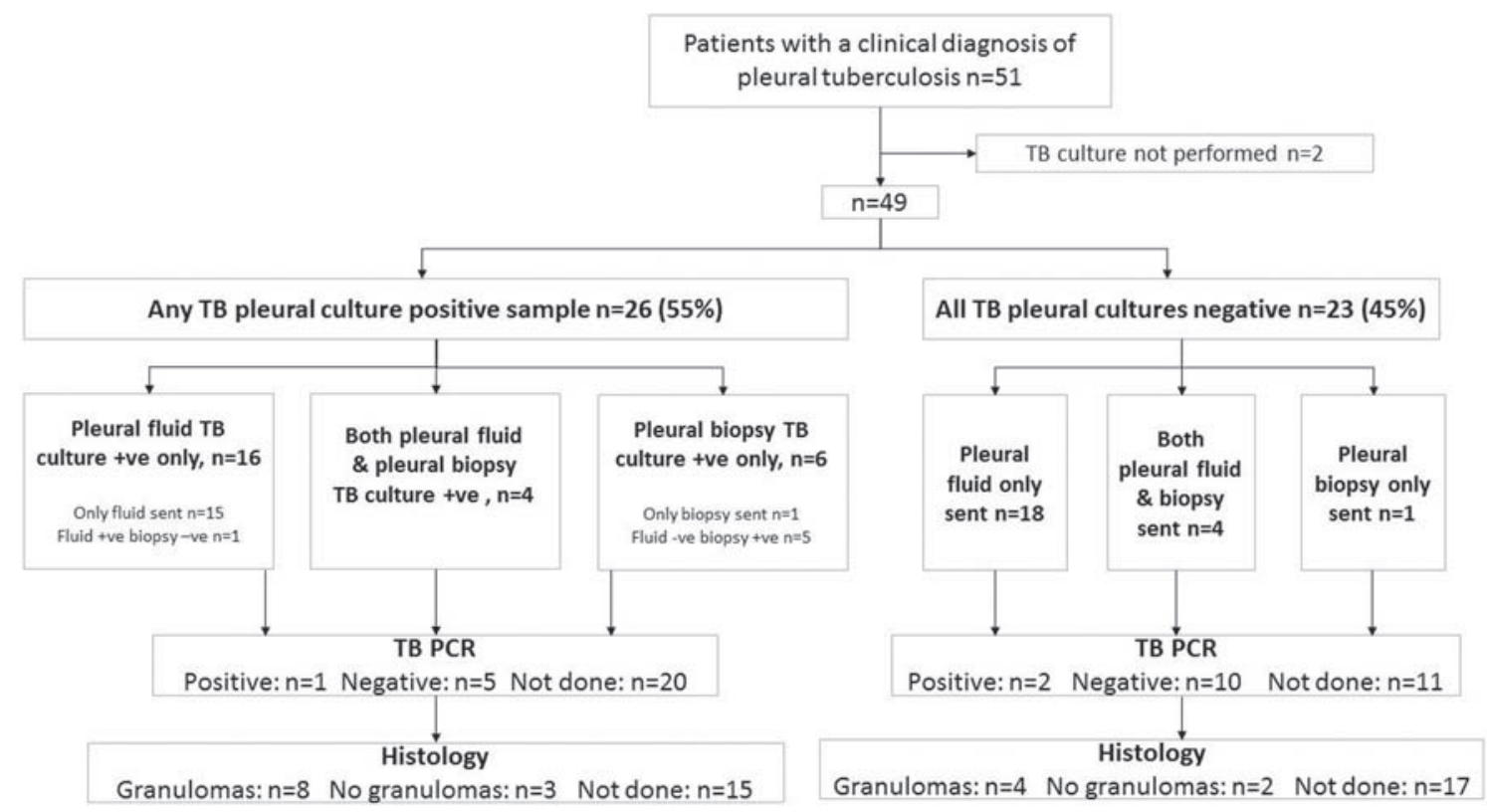


performed on $16 / 21$ samples. 63\% $(n=10)$ were culture positive. $4 / 6$ culture negative samples that were sent for histopathology also demonstrated granulomas. The combined yield of fluid and biopsy culture in addition to fluid PCR was 57\% in our cohort increasing to $65 \%$ when histology is included. 4 patients had a positive culture or PCR from another site. 15/ $51(29 \%)$ of patients were treated empirically without positive TB cultures, positive TB PCR results, or granulomas on biopsy.

Conclusions This analysis demonstrates that the characteristics of our cohort are similar to previously reported cohorts; that TB PCR of pleural fluid has provided some diagnostic benefits, but that there remains diagnostic uncertainty in a significant proportion of patients. New tools are required to improve diagnostic accuracy of this difficult disease and we propose that NICE-approved analysis of fluid for adenosine deaminase may be a useful additional investigation for this patient group.

\section{P6 OCULAR TUBERCULOSIS: A SURVEY OF UK CLINICAL PRACTICE}

${ }^{1} \mathrm{R}$ Hussain, ${ }^{2} \mathrm{H}$ Petrushkin, ${ }^{1} \mathrm{C}$ Barraclough, ${ }^{3} \mathrm{H}$ Kunst, ${ }^{2} \mathrm{C}$ Pravesio, ${ }^{1} \mathrm{VLC}$ White, ${ }^{3} \mathrm{JL}$ Potter ${ }^{1}$ Barts Health NHS Trust, London, UK; ${ }^{2}$ Moorfields Eye Hospital NHS Foundation Trust, London, UK; ${ }^{3}$ Queen Mary University London, London, UK

10.1136/thoraxjnl-2017-210983.148

Background Ophthalmic manifestations of tuberculosis (TB) are described as inflammatory events in one or both eyes involving the uvea, optic nerve or orbit. The diagnosis is almost always presumptive as mycobacterium are rarely cultured from ocular/periocular tissues. Ocular TB is rare in the developed world and there is a general lack of consensus regarding diagnosis and treatment duration. We surveyed UK specialists involved in the diagnosis and treatment of tuberculous uveitis to examine current clinical practice.

Method A previously validated survey based on two clinical cases (one more likely to have $\mathrm{TB}$, one less likely to have $\mathrm{TB}$ ) was used to examine diagnostic and treatment practices amongst consultants from three different specialities across different institutions in the UK: ophthalmologists with an interest in uveitis, respiratory, and infectious disease (ID) physicians with a TB interest.

Results Ten ophthalmologists, 24 ID and 29 respiratory physicians completed the survey. Responses varied greatly within the same specialty as well as between different specialities. For example, in a patient with chronic granulomatous panuveitis and a known TB risk factor, the pre-test likelihood of having ocular TB varied significantly within the groups: ophthalmologists (range 5\%-95\%), respiratory (range 20\%-99\%), ID (range 9\%-90\%). Similarly, for the same scenario, there was disagreement in the optimal duration of treatment. Whilst the majority of clinicians would treat for 6 months, 17 clinicians (24\%) - ophthalmologists [3], respiratory [5], ID [9] - would treat for longer than 6 months. All 10 ophthalmologists $(100 \%)$ would defer antibiotic treatment decisions to a TB specialist rather than initiate treatment themselves. All ID and respiratory physicians would screen for HIV if ocular TB was suspected, whereas only $6(60 \%)$ of ophthalmologists would. Conclusion Diagnosis of ocular $\mathrm{TB}$ is challenging due to lack of a gold standard test. Expert consensus is therefore important to ensure the right patients are treated appropriately. This is the first multidisciplinary survey within the UK capturing a spectrum of opinions regarding ocular TB. The Results highlight a lack of consensus both within and between different specialties in the field. An open dialogue between relevant stakeholders is key to harmonising diagnostic and treatment strategies.

\section{P7 TUBERCULOSIS IN DIFFERENTIAL DIAGNOSIS OF INTRATHORACIC LYMPHADENOPATHY IN A ENDEMIC COUNTRY - IS EBUS-TBNA A USEFUL TOOL?}

${ }^{1}$ LM Santos, ${ }^{2} \mathrm{M}$ Jacomelli, ${ }^{2}$ VR Figueiredo. ${ }^{1}$ Pneumology Unit, Hospitais da Universidade de Coimbra, Centro Hospitalar e Universitário de Coimbra, Coimbra, Portugal; ${ }^{2}$ Respiratory Endoscopy Service, Pulmonary Division of Heart Institute (InCor), Hospital das Clinicas, Faculdade de Medicina da Universidade de São Paulo, São Paulo, Brazil

\subsection{6/thoraxjnl-2017-210983.149}

Background Tuberculosis(TB) remains a global public health concern. ${ }^{1}$ It is one of intrathoracic lymphadenopathy(ITLN) numerous causes and must be taken into account in differential diagnosis, especially in endemic countries. ${ }^{2}$ Diagnostic yield of EBUS-TBNA for mediastinal and hilar TB has been reported to be $80 \% .^{2}$

Aim To determine the prevalence of TB in patients with ITLN undergoing EBUS-TBNA and calculate diagnostic yield.

Methods Retrospective study of all patients undergoing EBUSTBNA for ITLN, from August/2011 to March/2017. Clinical, laboratorial, histopathological and radiological data were assessed. We considered for diagnosis by EBUS-TBNA all cases with $\mathrm{TB}$ confirmed and granulomatous lymph node with $\mathrm{TB}$ confirmation by other methods.

Results 186 patients were included, mean age of 57 years $(\mathrm{SD}=14)$, 53.8\% male. Granulomatous disease was diagnosed in $29 \%(n=54), 42.6 \%(n=23)$ with cancer diagnosis or suspicion at the time of procedure. Sarcoidosis was diagnosed in $15.6 \%(n=29)$, mycobacteriosis in 9.7\% $(n=18)$, fungal infection in $2.7 \%(n=5)$ and other granulomatosis in $1 \%(n=2)$. Mycobacteriosis was due to M.tuberculosis in $88.9 \% \quad(n=16)$, M.kansasii in $5.55 \%(\mathrm{n}=1)$ and M.avium in $5.55 \%(\mathrm{n}=1)$. EBUS-TBNA established the diagnosis in 56.3\% $(\mathrm{n}=9)$ of TB cases: $25 \%(\mathrm{n}=4)$ caseous granuloma in cell block, $18.8 \%$ $(\mathrm{n}=3)$ positive acid-fast bacilli (AFB) and $12.5 \% \quad(\mathrm{n}=2) \mathrm{M}$. Tuberculosis in aspirated sample culture. In 37.5\% $(n=6)$ cell block from EBUS-TBNA showed granulomas but definite diagnosis was made by presence of caseous granuloma in biopsy $(12.5 \%, \mathrm{n}=2)$ or positive $\mathrm{AFB}(6.25 \%, \mathrm{n}=1)$ in other organs, positive PCR of bronchoalveolar lavage in $6.25 \%(n=1)$ or pleural fluid in $6.25 \%(\mathrm{n}=1)$, and sputum culture in $6.25 \%$ $(n=1)$. One patient needed mediastinoscopy. 25\% $(n=4)$ of patients diagnosed with TB had cancer and $31.6 \%(n=5)$ was submitted to EBUS-TBNA for cancer suspicion. In our setting, the prevalence of $\mathrm{TB}$ was $8.6 \%$ and the diagnostic yield of EBUS-TBNA was $77.7 \%$.

Conclusions Our study showed granulomatosis in $29 \%$ of patients with $\mathrm{TB}$ as second most frequent cause.Brazil belongs to the five countries that collectively account for about $50 \%$ of the world TB cases ${ }^{1}$ and, despite lymphadenopathy is not a major form of TB, our study emphasises its importance in differential diagnosis of ITLN in endemic countries. EBUS-TBNA showed to be a useful diagnostic tool.

\section{REFERENCES}

1. World Health Organisation. Global tuberculosis control: WHO report 2016. http:// apps. who.int/iris/bitstream/10665/250441/1/9789241565394-eng.pdf?ua=1 